\title{
Robust Adaptive Tracking Control based on State Feedback Controller with Integrator Terms for Elastic Joint Robots with Uncertain Parameters
}

\author{
Luc Le-Tien, and Alin Albu-Schäffer, Fellow, IEEE
}

\begin{abstract}
This paper addresses a robust adaptive control scheme based on a cascaded structure with a full state feedback controller with integrator terms as inner control loop and computed torque as outer control loop for flexible joint robots. Together with integrator effect, the adaptive control law can enhance position accuracy under uncertainties of the robot model, especially, the high friction caused by Harmonic-Drive with high gear ratio. In this paper the adaptive friction compensation is designed based on the LuGre friction model, which exhibits some advantages compared to the static friction model (e.g. no chattering effect at zero motor velocity). Furthermore, structural oscillations of the link side can be effectively damped by using joint torque feedback in the state feedback controller. So, the proposed adaptive control approach can simultaneously provide high control performance both in terms of the dynamic behavior and the position accuracy. Global asymptotic tracking is achieved for the complete controlled system. The system stability is derived using Lyapunov approaches and Barbalat's lemma. Experimental results validate practical efficiency of the approach.
\end{abstract}

Index Terms-Adaptive control, tracking control, state feedback control, cascaded control, flexible joint robots

\section{INTRODUCTION}

In order to increase the payload/weight ratio, elastic joint robots use Harmonic-Drive ${ }^{\circledR}$ gears with high ratio $(1: 100$ or higher). But, high gear ratio causes high motor friction and high robot elasticity, which are challenging problems for robot control. In addition, the dynamics parameters of the robot (especially, friction parameters) can vary with time or with temperature, motivating the adaptive control development of this paper.

Most tracking control of flexible joint robots was developed based on the assumption that the link position is directly measured and that its higher derivatives can be computed. Different control strategies have been dealt in great detail in the literature, e.g. singular perturbation [1], [2], feedback input-output linearization [3], [4], cascaded control [5], [6], backstepping [7], [8], and passivity-based approaches in [9], [10], using either full or partial state feedback. A combination of a partial state feedback linearization technique and a backstepping design method was introduced in [11] to reach a global output tracking control. Furthermore, in [12], [13] nonlinear observer based controllers were proposed for flexible joint robots.

Instead of measuring robot joint positions, link torque sensors can be alternatively used to feedback the link side state

Luc Le-Tien is with the Robotics and Mechatronics Center, German Aerospace Center (DLR), Oberpfaffenhofen, 82234 Wessling, Germany Luc. Le-Tiendalr.de.

Alin Albu-Schäffer is with the Robotics and Mechatronics Center, German Aerospace Center (DLR), Oberpfaffenhofen, D-82234 Wessling, Germany and with the Department of Informatics, Technical University of Munich, 85748 Garching, Germany Alin.Albu-Schaefferedlr.de.

Manuscript received January 19, 2017; revised April 19, 2017. to eliminate link oscillations caused by high joint elasticity. In [14] a set-point controller with full state feedback (motor position, link side torque, their derivatives) was proposed in order to reduce robot vibration and hence the transient performance can be improved through feedback of the link torques. This controller is very robust against uncertainties of the robot dynamics parameters due to its simple structure, but it does not fulfill high accuracy requirements. For tracking control using link torque feedback, in [5] a cascaded controller was developed which consists of two control loops, a motion controller as outer control loop and a torque controller as inner control loop, whereas the robust cascaded controller in [15] uses a motion controller and a state feedback controller with full state feedback (motor position, link side torque, their derivatives) as outer and inner control loop respectively.

Furthermore, in order to consider uncertainties of the robot parameters or varying parameters, some adaptive control schemes were introduced in [7], [5]. Based on cascaded analysis, in [16] a robust adaptive control scheme was introduced by using the sliding mode technique. In [17], [18] the adaptive control scheme is extended including adaptive friction compensation for flexible joint robots, which however takes only static friction into account, without modeling dynamical effects. The problem of adaptive friction compensation based on a LuGre dynamic friction model was treated for rigid robot in [19], [20], [21].

Most previous adaptive tracking control schemes can provide high position accuracy, but can hardly achieve a good dynamic behavior. In order to retain both the advantages of robust and adaptive controls, in this paper a robust adaptive controller was proposed based on a cascaded scheme like in [15], but the inner control loop is a robust full state feedback controller with integrator terms (integrated motor position, motor position, motor velocity, link torque, derivative of the link torque) in order to increase position accuracy. This approach includes adaptive friction compensation based on the LuGre friction model. It can achieve a good transient behavior and a high position accuracy, and simultaneously tolerates time-varying parameters. In an analogous manner to [15], [22] global asymptotic tracking is achieved. The system stability is derived using Lyapunov approaches and Barbalat's lemma. The resulting robust adaptive controller is experimentally verified and compared with the conventional PID controller and the corresponding model based controller for the DLR (German Aerospace Center) medical robot [23] (see Figure 1), which is equipped with motor position sensors and link torque sensors used for robot motion control.

The remainder of this paper is organized as follows. Section II introduces the dynamic robot model. In Sec. III the cascaded control approach consisting of the computed torque 


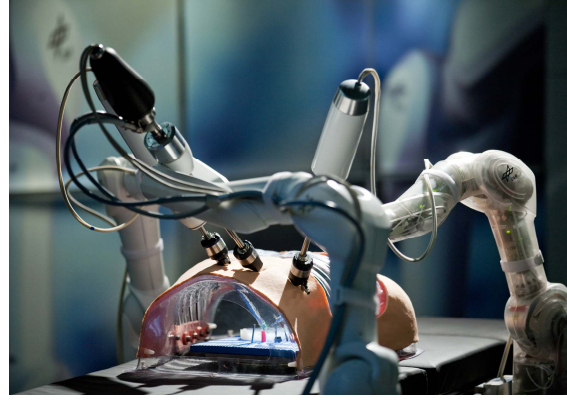

Figure 1. The DLR medical robots used in minimally invasive surgery.

controller and the state feedback controller with integrator terms using model-based friction compensation is proposed for flexible joint robots and the stability of the controlled system is analyzed. Sec. IV introduces an extension of this control structure to a robust adaptive control approach and presents its stability analysis. Finally, the obtained performance is verified by experiments reported in Sec. V.

\section{MODELING THE ROBOT DYNAMICS}

\section{A. Robot Model}

For a flexible joint robot with $n$ rotary joints its simplified dynamics [1], [24] is described by

$$
\begin{aligned}
u & =J \ddot{\theta}+\tau+\tau_{f} \\
\tau & =M(q) \ddot{q}+C(q, \dot{q}) \dot{q}+g(q) .
\end{aligned}
$$

Therein, $q \in \mathbb{R}^{n}$ and $\theta \in \mathbb{R}^{n}$ are the link and motor angles, respectively. $\tau_{f} \in \mathbb{R}^{n}$ is the friction torque. The control input is the motor torque $u \in \mathbb{R}^{n}$. The motor inertia matrix $J \in$ $\mathbb{R}^{n \times n}$ is diagonal and positive definite. The transmission torque between motor and link dynamics $\tau \in \mathbb{R}^{n}$ is modeled as a linear function of the motor and the link position

$$
\tau=K(\theta-q)
$$

and is measured by strain gauge based torque sensors. The joint stiffness matrix $K \in \mathbb{R}^{n \times n}$ is diagonal and positive definite. Furthermore, $M(q) \in \mathbb{R}^{n \times n}$ is the mass matrix, $C(q, \dot{q}) \in \mathbb{R}^{n \times n}$ the centrifugal and Coriolis matrix, and $g(q) \in \mathbb{R}^{n}$ the gravity vector of the rigid body model.

Finally, in order to facilitate the controller design and the stability analysis, the following two properties are used

P.1: $\quad$ The mass matrix $M(q)$ is symmetric and positive definite $M(q)=M^{T}(q)$ and satisfies

$$
\lambda_{m} \leq\|M(q)\| \leq \lambda_{M}
$$

with $\lambda_{m}, \lambda_{M}$ being the maximum and minimum eigenvalues respectively.

P.2: $\quad$ The matrix $\dot{M}(q)-2 C(q, \dot{q})$ is skew symmetric and

$$
x^{T}(\dot{M}(q)-2 C(q, \dot{q})) x=0, \quad \forall x, q, \dot{q} \in \mathbb{R}^{n} .
$$

In Addition, the following assumptions are made

A.1: The motor position $\theta$ and the link side torque $\tau$ are directly measured and can be used for feedback control.

A.2: $\quad$ The desired link position $q_{d}(t) \in C^{4}$ is bounded.

\section{B. LuGre Friction Model}

For the friction torque a dynamic friction model (LuGre friction model [25], [15]) is chosen as

$$
\tau_{f}=\sigma_{0} z+\sigma_{1} \dot{z}+f_{v} \dot{\theta}
$$

with the inner dynamics of the friction

$$
\left\{\begin{array}{l}
\dot{z}=\dot{\theta}-\frac{|\dot{\theta}|}{h_{z}} \sigma_{0} z \\
h_{z}=f_{c}+f_{l}|\tau| .
\end{array}\right.
$$

Therein, $\tau$ is the measured link torque. $\sigma_{0}$ and $\sigma_{1}$ are stiffness and damping coefficients of the LuGre friction model. $h_{z}$ describes effects of the static friction [25]. $f_{c}, f_{v}$ and $f_{l}$ represent the Coulomb, viscous and load dependent coefficients of the friction torque, respectively.

To design an adaptive friction compensator we will reformulate the inner dynamics (6) by setting

$$
\dot{z}=\dot{\theta}-\Psi z
$$

with the positive definite and diagonal matrix

$$
\Psi=\operatorname{diag}\left(\sigma_{0_{1}} \frac{\left|\dot{\theta}_{1}\right|}{h_{z_{1}}}, \ldots, \sigma_{0_{n}} \frac{\left|\dot{\theta}_{n}\right|}{h_{z_{n}}}\right) .
$$

Now the friction model (5) can be rewritten by

$$
\tau_{f}=\sigma_{0} z-\sigma_{1} \Psi z+\sigma_{2} \dot{\theta}
$$

with $\sigma_{2}=\sigma_{1}+f_{v}$.

Furthermore, let us redefine the LuGre friction model (9) as a friction model with two inner independent states $z_{0}, z_{1}$

$$
\tau_{f}=\Phi_{0} z_{0}-\Phi_{1} \Psi z_{1}+\Phi_{2} \dot{\theta},
$$

and with two inner independent friction dynamics

$$
\left\{\begin{array}{l}
\dot{z}_{0}=\dot{\theta}-\Psi z_{0} \\
\dot{z}_{1}=\dot{\theta}-\Psi z_{1} .
\end{array}\right.
$$

All matrices $\Phi_{0}, \Phi_{1}, \Phi_{2} \in \mathbb{R}^{n \times n}$ are positive definite and diagonal. For the adaptive friction compensation these parameters and simultaneously the inner states $z_{0}, z_{1}$ have to be estimated online during trajectory tracking of the robot.

\section{CASCADED CONTROL WITH KNOWN DYNAMICS PARAMETERS}

The new control law is designed based on the cascaded structure, for which two control loops are used. The outer control loop computes the desired values (e.g. the desired motor position and the desired link torque) for the inner control loop. In a different way from [15], in the inner control loop a state feedback controller with integrator terms is used to compute the desired motor torque, which should increase the tracking position accuracy of the robot.

Now let us denote the desired motor position and the desired link position as $\theta_{d}$ and $q_{d}$, respectively. According to these variables the desired link torque can be defined by

$$
\tau_{d}=K\left(\theta_{d}-q_{d}\right) .
$$

Together with (3) this leads to the link torque error

$$
e_{\tau}=\tau_{d}-\tau=K\left(e_{\theta}-e_{q}\right)
$$

with $e_{\theta}$ and $e_{q}$ being the position tracking errors of the motor side and the link side

$$
\left\{\begin{array}{l}
e_{\theta}=\theta_{d}-\theta \\
e_{q}=q_{d}-q .
\end{array}\right.
$$


A. Motor Motion Control based on a State Feedback Controller with Integrator Terms

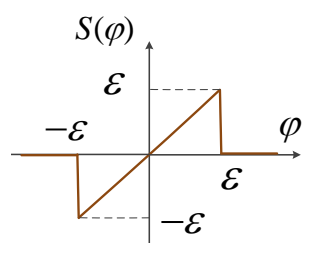

Figure 2. $S(\varphi)$ function.

Let $\Lambda$ be a positive definite and diagonal matrix, and define

$$
\left\{\begin{array}{l}
v_{\theta}=\dot{\theta}_{d}+\Lambda e_{\theta} \\
s_{\theta}=v_{\theta}-\dot{\theta}=\dot{e}_{\theta}+\Lambda e_{\theta} .
\end{array}\right.
$$

In this inner control loop, in order to ensure that the link position $q$ converges to the desired link position $q_{d}$, a state feedback controller with integrator terms consisting of full states (integrated motor position, motor position, motor velocity, link torque, derivative of the link torque) is proposed as

$$
\begin{aligned}
& u=J \dot{v}_{\theta}+K_{I} S(\varphi(t))+K_{P} e_{\theta}+K_{D} \dot{e}_{\theta} \\
& +K_{T} K^{-1} e_{\tau}+K_{S} K^{-1} \dot{e}_{\tau}+\tau_{d}+\tau_{f}
\end{aligned}
$$

where $\varepsilon$ is a positive constant. Obviously, $S(\varphi)$ is a bounded function.

Figure 2 depicts the definition of the bounded function $S(\varphi)$. In the following, we assume that the initial $s_{\theta}(t=0)=$ 0 . Then, the time derivative of $S(\varphi)$ becomes

$$
\dot{S}(\varphi(t))=\frac{\partial S}{\partial \varphi} \frac{\partial \varphi}{\partial t}= \begin{cases}s_{\theta}(t) & \text { if }|\varphi(t)| \leq \varepsilon \\ 0 & \text { if }|\varphi(t)|>\varepsilon .\end{cases}
$$

Furthermore, all gain matrices $K_{I}, K_{P}, K_{D}, K_{T}$ and $K_{S} \in \mathbb{R}^{n \times n}$ are positive definite and diagonal. The friction compensation term $\tau_{f}$ is computed by using the LuGre friction model (5).

By inserting the control law (16) into (1) and then using the definition (13), one gets the closed-loop motor dynamics

$$
\begin{aligned}
& J \dot{s}_{\theta}+K_{I} S(\varphi)+K_{P} e_{\theta}+K_{D} \dot{e}_{\theta} \\
& +A\left(e_{\theta}-e_{q}\right)+K_{S}\left(\dot{e}_{\theta}-\dot{e}_{q}\right)=0 .
\end{aligned}
$$

with $A$ being positive definite and diagonal matrix and

$$
A=K_{T}+K .
$$

The equation (19) will be used for stability analysis in Sec. III-C.

In order to use the control law (16), the desired link torque as well as the desired motor position and their derivatives have to be computed in the next section.

Remark 1: The design of controllers for robot manipulators has to deal with the problem of bounded torques in the externally applied control torque signals, because motors can provide finite torques only. Since the integral term generates energy in the system, the bounded function $S(\varphi)$ in (17) is required to bound control torques and guaranty energy dissipation for the controlled system. As consequence the position errors of the system converge to zero according to Barbalat's lemma (see more proof of stability in Sec. III.A). Due to modeling errors and variable loads, the gravitational and the frictional torque are subjected to uncertainties that require that the applied control torque should be sufficiently large to counteract them at the desired position. Therefore, the threshold $\varepsilon$ of the function $S(\varphi)$ could be chosen so that $\varepsilon>K_{I}^{-1}\left(\sup _{\dot{\theta}, \tau \in \mathbb{R}^{n}}\left|\tau_{f_{i}}\right|+\sup _{q \in \mathbb{R}^{n}}\left|g_{i}(q)\right|\right)$ for $i=1 . . n$. In practice $\varepsilon$ was chosen big enough so that the integral action is always active for all time.

\section{B. Computed Torque Control}

Further let us define

$$
\left\{\begin{array}{l}
v_{q}=\dot{q}_{d}+\Lambda e_{q} \\
s_{q}=v_{q}-\dot{q}=\dot{e}_{q}+\Lambda e_{q} .
\end{array}\right.
$$

In this outer control loop the desired link torque and the desired motor position are computed. Their appropriate derivatives can be obtained through numerical differentiation. Therefore, the desired link torque $\tau_{d}$ for the control law (16) can be generated based on using the rigid body dynamics [26] and is given by

$$
\tau_{d}=M(q) \dot{v}_{q}+C(q, \dot{q}) v_{q}+g(q)+K_{q} s_{q},
$$

where the matrix $K_{q}$ is positive definite and diagonal. It is noticed that this additional control damping term enables to enhance system control performance in terms of the dynamic behavior.

Hence, the desired motor position is determined from the definition (12)

$$
\theta_{d}=q_{d}+K^{-1}\left[M(q) \dot{v}_{q}+C(q, \dot{q}) v_{q}+g(q)+K_{q} s_{q}\right] .
$$

Simultaneously, for this proposed desired link torque one can obtain the closed-loop link dynamics by inserting (22) and (2) into (13)

$$
e_{\tau}=\tau_{d}-\tau=M(q) \dot{s}_{q}+\left(C(q, \dot{q})+K_{q}\right) s_{q} .
$$

Remark 2: According to (3) the link position can be determined from the measured motor position and the measured link torque by $q=\theta-K^{-1} \tau$.

Remark 3: The motor velocity and the derivative of the link torque can be approximated by the first numerical difference. With the high sampling rate $(3 \mathrm{kHz}$ for the DLR medical robots), this approximation is accurate enough for motion control purposes. Then it follows $\dot{q}=\dot{\theta}-K^{-1} \dot{\tau}$.

Remark 4: According to the control law (16) the derivative of the desired link torque $\dot{\tau}_{d}$ and the desired motor velocity $\dot{\theta}_{d}$ (as well as the motor acceleration $\ddot{\theta}_{d}$ ) are needed. Deriving (22), (23) uses the link acceleration $\ddot{q}$, which can be computed by using the link dynamics (2) instead of numeric differentiation. Therefore, the control law (16) is only depended on the measured motor position, the measured link torque and their fist derivatives, $\dot{\theta}_{d}\left(q_{d}, \dot{q}_{d}, \ddot{q}_{d}, q_{d}^{(3)}, \theta, \dot{\theta}, \tau, \dot{\tau}\right)$, $\dot{\tau}_{d}\left(q_{d}, \dot{q}_{d}, \ddot{q}_{d}, q_{d}^{(3)}, \theta, \dot{\theta}, \tau, \dot{\tau}\right), \ddot{\theta}_{d}\left(q_{d}, \dot{q}_{d}, \ddot{q}_{d}, q_{d}^{(3)}, q_{d}^{(4)}, \theta, \dot{\theta}, \tau, \dot{\tau}\right)$. 


\section{Stability Analysis}

Theorem 1: Consider the robot dynamic system (1) and (2) satisfying assumption that the robot parameters are known. With the motion controller (16) and the joint torque controller (22) the system achieves global asymptotic convergence $\left\{\lim _{t \rightarrow \infty} e_{\theta}=0, \lim _{t \rightarrow \infty} \dot{e}_{\theta}=0, \lim _{t \rightarrow \infty} e_{q}=\right.$ $\left.0, \lim _{t \rightarrow \infty} \dot{e}_{q}=0\right\}$ whenever the following condition is satisfied

$$
K_{q}>\frac{1}{4} K A^{-1} K_{S}^{2}\left(\Lambda K_{P}^{-1}+\left(K_{D}+K_{S}\right)^{-1}\right) .
$$

Proof: For stability analysis the following Lyapunov function candidate is chosen

$$
\begin{aligned}
& V(x)=\frac{1}{2} s_{\theta}^{T} K A^{-1} J s_{\theta}+\frac{1}{2}\left(e_{\theta}-e_{q}\right)^{T} K\left(e_{\theta}-e_{q}\right) \\
& +\frac{1}{2} s_{q}^{T} M(q) s_{q}+\frac{1}{2} e_{\theta}^{T} K A^{-1}\left(K_{P}+\Lambda K_{D}+\Lambda K_{S}\right) e_{\theta} \\
& +e_{q}^{T} K_{q} \Lambda e_{q}+\frac{1}{2} S(\varphi)^{T} K A^{-1} K_{I} S(\varphi) \\
& +\int_{0}^{t}\left(e_{\theta}-e_{q}\right)^{T} K \Lambda\left(e_{\theta}-e_{q}\right) d t
\end{aligned}
$$

with $x=\left\{S(\varphi), \int e_{\theta}, e_{\theta}, \dot{e}_{\theta}, \int e_{q}, e_{q}, \dot{e}_{q}\right\}$. It is noticed that all the gain matrices as well the stiffness matrix and parameter matrix $A$ are diagonal and positive definite. Hence, the function $V$ is positive definite. Furthermore, because of the boundedness of the gain matrices and the mass matrix $M(q)$ from property (P.1), $V$ is also bounded by $\alpha\|x\|^{2} \leq V \leq \beta\|x\|^{2}$.

Then, the derivative of the function $V$ along the trajectory, using equations (19) and (24), leads to

$$
\begin{aligned}
& \dot{V}=s_{\theta}{ }^{T} K A^{-1} J \dot{s}_{\theta}+\left(e_{\theta}-e_{q}\right)^{T} K\left(\dot{e}_{\theta}-\dot{e}_{q}\right)+\frac{1}{2} s_{q}{ }^{T} \dot{M}(q) s_{q} \\
& +s_{q}^{T} M(q) \dot{s}_{q}+e_{\theta}^{T} K A^{-1}\left(K_{P}+\Lambda K_{D}+\Lambda K_{S}\right) \dot{e}_{\theta} \\
& +2 e_{q}^{T} K_{q} \Lambda \dot{e}_{q}+\dot{S}(\varphi)^{T} K A^{-1} K_{I} S(\varphi) \\
& +\left(e_{\theta}-e_{q}\right)^{T} K \Lambda\left(e_{\theta}-e_{q}\right) \equiv \dot{V}_{1}+\dot{V}_{2}
\end{aligned}
$$

with

$$
\begin{aligned}
\dot{V}_{1}= & -s_{\theta}{ }^{T} K A^{-1} K_{I} S(\varphi)+\dot{S}(\varphi)^{T} K A^{-1} K_{I} S(\varphi) \\
\dot{V}_{2}= & s_{\theta}{ }^{T} K A^{-1}\left[-K_{P} e_{\theta}-K_{D} \dot{e}_{\theta}-A\left(e_{\theta}-e_{q}\right)\right. \\
& \left.-K_{S}\left(\dot{e}_{\theta}-\dot{e}_{q}\right)\right]+\frac{1}{2} s_{q}{ }^{T} \dot{M}(q) s_{q}+s_{q}{ }^{T}\left[e_{\tau}\right. \\
& \left.-\left(C(q, \dot{q})+K_{q}\right) s_{q}\right]+\left(e_{\theta}-e_{q}\right)^{T} K\left(\dot{e}_{\theta}-\dot{e}_{q}\right) \\
& +e_{\theta}{ }^{T} K A^{-1}\left(K_{P}+\Lambda K_{D}+\Lambda K_{S}\right) \dot{e}_{\theta} \\
& +2 e_{q}{ }^{T} K_{q} \Lambda \dot{e}_{q}+\left(e_{\theta}-e_{q}\right)^{T} K \Lambda\left(e_{\theta}-e_{q}\right) .
\end{aligned}
$$

Using the properties of the bounded function $S(\varphi)$ in (17), (18) obviously results in

$$
\begin{aligned}
\dot{V}_{1} & = \begin{cases}-s_{\theta}{ }^{T} K A^{-1} K_{I} S(\varphi)+s_{\theta}^{T} K A^{-1} K_{I} S(\varphi) \\
0 & \text { if }|\varphi| \leq \varepsilon\end{cases} \\
& =0 \quad \forall \varphi(t) .
\end{aligned}
$$

Hence $\dot{V}$ is semi-negative definite, when $\dot{V}_{2}$ is semi-negative definite. Furthermore, by simplifying $\dot{V}_{2}$ in (28) using (13) and the property (P.2) one obtains

$$
\begin{aligned}
& \dot{V}_{2}\left(e_{\theta}, \dot{e}_{\theta}, e_{q}, \dot{e}_{q}\right)=-e_{\theta}^{T} \Lambda K A^{-1} K_{P} e_{\theta} \\
& \quad-\dot{e}_{\theta}^{T} K A^{-1}\left(K_{D}+K_{S}\right) \dot{e}_{\theta}+e_{\theta}^{T} \Lambda K A^{-1} K_{S} \dot{e}_{q} \\
& +\dot{e}_{\theta}^{T} K A^{-1} K_{S} \dot{e}_{q}-e_{q}^{T} \Lambda K_{q} \Lambda e_{q}-\dot{e}_{q}^{T} K_{q} \dot{e}_{q} \\
& :=-\left[\begin{array}{llll}
e_{\theta}^{T} & \dot{e}_{\theta}^{T} & e_{q}^{T} & \dot{e}_{q}^{T}
\end{array}\right] H\left[\begin{array}{c}
e_{\theta} \\
\dot{e}_{\theta} \\
e_{q} \\
\dot{e}_{q}
\end{array}\right]
\end{aligned}
$$

with the symmetric Hessian matrix

$H=\left[\begin{array}{cccc}\Lambda K A^{-1} K_{P} & 0 & 0 & -\frac{1}{2} \Lambda K A^{-1} K_{S} \\ 0 & K A^{-1}\left(K_{D}+K_{S}\right) & 0 & -\frac{1}{2} K A^{-1} K_{S} \\ 0 & 0 & \Lambda K_{q} \Lambda & 0 \\ -\frac{1}{2} \Lambda K A^{-1} K_{S} & -\frac{1}{2} K A^{-1} K_{S} & 0 & K_{q}\end{array}\right]$.

The zero matrix is denoted by $0 \in \mathbb{R}^{n \times n}$. This Hessian matrix $H$ can be divided by

$$
H=\left[\begin{array}{ll}
H_{11} & H_{12} \\
H_{12}^{T} & H_{22}
\end{array}\right]
$$

with $H_{11}, H_{12}$ and $H_{22} \in \mathbb{R}^{2 n \times 2 n}$ being sub-matrices. Now, $\dot{V}_{2}$ is negative definite, when $H$ is positive definite. From the lemma "positive definite symmetric matrix" [28], the following two inequality conditions should be fulfilled

$$
\begin{aligned}
& H_{11}>0 \\
& H_{22}>H_{12}^{T}\left(H_{11}\right)^{-1} H_{12} .
\end{aligned}
$$

Condition (32) is always fulfilled with positive definite matrices $\Lambda, K, A, K_{P}, K_{D}$ and $K_{S}$. Further, the Schur complement of the matrix $H$ is given by

$$
\begin{aligned}
H_{s} & =H_{22}-H_{12}^{T}\left(H_{11}\right)^{-1} H_{12} \\
& =\left[\begin{array}{cc}
\Lambda K_{q} \Lambda & 0 \\
0 & K_{q}-\frac{1}{4} K A^{-1} K_{S}^{2}\left(\Lambda K_{P}^{-1}+\left(K_{D}+K_{S}\right)^{-1}\right)
\end{array}\right] .
\end{aligned}
$$

Obviously, condition (33) is fulfilled when $H_{s}$ is positive definite, or, equivalently, the condition (25) is fulfilled. Then $\dot{V}_{2}\left(e_{\theta}, \dot{e}_{\theta}, e_{q}, \dot{e}_{q}\right)$ is negative semi-definite with the condition (25) and therefore $\{\dot{V} \leq 0 \mid \forall x\}$.

The equilibrium $x=0$ is stable in the sense of Lyapunov, because $\{V>0 \mid \forall x \neq 0\},\{\dot{V} \leq 0 \mid \forall x\}$ and $\{\dot{V}<0 \mid \forall x \neq$ $0\} . S(\varphi), e_{\theta}, \dot{e}_{\theta}, e_{q}$ and $\dot{e}_{q}$ are bounded and belong to $L_{2}$, and from (15), (19), (21) and (24) one has

$$
\begin{gathered}
\ddot{e}_{\theta}=-\Lambda \dot{e}_{\theta}-J^{-1}\left(K_{I} S(\varphi)+K_{P} e_{\theta}+K_{D} \dot{e}_{\theta}\right. \\
\left.+A\left(e_{\theta}-e_{q}\right)+K_{S}\left(\dot{e}_{\theta}-\dot{e}_{q}\right)\right) \\
\ddot{e}_{q}=-\Lambda \dot{e}_{q}+M(q)^{-1}\left(K\left(\dot{e}_{\theta}-\dot{e}_{q}\right)\right. \\
\left.\quad-\left(C(q, \dot{q})+K_{q}\right)\left(\dot{e}_{q}+\Lambda e_{q}\right)\right) .
\end{gathered}
$$

Evidently, $\ddot{e}_{q}$ and $\ddot{e}_{\theta}$ are bounded as well because all variables in the right hand side of the about two equations are bounded. This yields that the derivative of $\dot{V}$ is bounded, according to Barbalat's lemma [26] the function $\dot{V}$ is uniformly continuous, and $\dot{V} \rightarrow 0$ as $t \rightarrow \infty$. This means that the system errors converge to the zero equilibrium asymptotically, $\left\{\lim _{t \rightarrow \infty} e_{\theta}=\right.$ $\left.0, \lim _{t \rightarrow \infty} \dot{e}_{\theta}=0, \lim _{t \rightarrow \infty} e_{q}=0, \lim _{t \rightarrow \infty} \dot{e}_{q}=0\right\}$, or, equivalently, $\left\{\theta \rightarrow \theta_{d}, q \rightarrow q_{d}\right\}$. This completes the proof of the theorem 1 . 


\section{ADAPTIVE CONTROL WITH UNKNOWN DYNAMICS PARAMETERS}

In practice, all parameters can be unknown in both link and motor dynamics including friction. In order to improve the position accuracy while keeping the cascaded control structure with the state feedback controller with integrator terms, in this section the cascaded control structure is extended to an adaptive control approach including adaptive friction compensation.

\section{A. Adaptive Motor Motion Control}

In order to achieve better position accuracy we propose a new adaptive control law with adaptive friction compensation

$$
\begin{gathered}
\qquad \begin{array}{c}
\widehat{J} \dot{\hat{v}}_{\theta}+K_{I} S(\hat{\varphi})+K_{P} \hat{e}_{\theta}+K_{D} \dot{\hat{e}}_{\theta} \\
+K_{T} K^{-1} \hat{e}_{\tau}+K_{S} K^{-1} \dot{\hat{e}}_{\tau}+\hat{\tau}_{d}+\hat{\tau}_{f}
\end{array} \\
\text { with } \begin{cases}\hat{e}_{\theta}=\hat{\theta}_{d}-\theta, & \hat{v}_{\theta}=\dot{\hat{\theta}}_{d}+\Lambda \hat{e}_{\theta} \\
\hat{s}_{\theta}=\dot{\hat{e}}_{\theta}+\Lambda \hat{e}_{\theta}, & \hat{e}_{\tau}=\hat{\tau}_{d}-\tau\end{cases} \\
\text { and } S(\hat{\varphi})=\left\{\begin{array}{ll}
\hat{\varphi} & \text { if }|\hat{\varphi}| \leq \varepsilon \\
0 & \text { if }|\hat{\varphi}|>\varepsilon
\end{array}, \quad \hat{\varphi}(t)=\int_{0}^{t} \hat{s}_{\theta} d t\right.
\end{gathered}
$$

where $\widehat{J}, \hat{\theta}_{d}, \hat{\tau}_{d}$ and $\hat{\tau}_{f}$ are the estimate of the motor inertia, the desired motor position, the desired link torque and the friction torque respectively. The estimates of the desired motor position, the desired link torque are computed in next subsection. For the adaptive friction compensation the estimate of the friction torque is chosen by

$$
\hat{\tau}_{f}=\hat{\Phi}_{0} \hat{z}_{0}-\hat{\Phi}_{1} \Psi \hat{z}_{1}+\hat{\Phi}_{2} \hat{v}_{\theta} .
$$

Hereby, $\hat{z}_{0}, \hat{z}_{1}$ and $\hat{\Phi}_{0}, \hat{\Phi}_{1}, \hat{\Phi}_{2}$ are the estimates of the inner states and the friction parameters respectively. The observed friction dynamics can be given by using the observers [27]

$$
\left\{\begin{array}{l}
\dot{\hat{z}}_{0}=\dot{\theta}-\Psi \hat{z}_{0}+\hat{s}_{\theta} \\
\dot{\hat{z}}_{1}=\dot{\theta}-\Psi \hat{z}_{1}-\hat{s}_{\theta} .
\end{array}\right.
$$

By setting $\tilde{z}_{0}=z_{0}-\hat{z}_{0}, \tilde{z}_{1}=z_{1}-\hat{z}_{1}$ and together with the friction dynamics (11) the closed loop friction dynamics result in

$$
\left\{\begin{array}{l}
\dot{\tilde{z}}_{0}=-\Psi \tilde{z}_{0}-\hat{s}_{\theta} \\
\dot{\tilde{z}}_{1}=-\Psi \tilde{z}_{1}+\hat{s}_{\theta} .
\end{array}\right.
$$

Furthermore, the relation between the desired motor position and the desired link torque is defined by

$$
\hat{\tau}_{d}=K\left(\hat{\theta}_{d}-q_{d}\right) .
$$

Together with (3) it follows $\hat{e}_{\tau}=K\left(\hat{e}_{\theta}-e_{q}\right)$. Then, inserting the control law (34) into the motor dynamics (1) and using (10), (37), (20) lead to the new closed loop equation

$$
\begin{aligned}
& J \dot{\hat{s}}_{\theta}+K_{I} S(\hat{\varphi})+ K_{P} \hat{e}_{\theta}+K_{D} \dot{\hat{e}}_{\theta}+A\left(\hat{e}_{\theta}-e_{q}\right) \\
&+K_{S}\left(\dot{\hat{e}}_{\theta}-\dot{e}_{q}\right)-\tilde{\Phi}_{0} \hat{z}_{0}-\Phi_{0} \tilde{z}_{0}+\tilde{\Phi}_{1} \Psi \hat{z}_{1} \\
&+\Phi_{1} \Psi \tilde{z}_{1}+\Phi_{2} \hat{s}_{\theta}-\tilde{\Phi}_{2} \hat{v}_{\theta}=\tilde{J}_{\hat{v}} .
\end{aligned}
$$

with $\tilde{\Phi}_{0}=\Phi_{0}-\hat{\Phi}_{0}, \tilde{\Phi}_{1}=\Phi_{1}-\hat{\Phi}_{1}, \tilde{\Phi}_{2}=\Phi_{2}-\hat{\Phi}_{2}$, and $\widetilde{J}=J-\widehat{J}$.

Because of the parameter matrices $\widetilde{J}, \tilde{\Phi}_{0}, \tilde{\Phi}_{1}, \tilde{\Phi}_{2}$ being diagonal, they can be rewritten as vectors of their diagonal elements. Hence, let us define the new vectors $\widetilde{\gamma}_{\theta}, \tilde{\gamma}_{0}, \tilde{\gamma}_{1}, \tilde{\gamma}_{2} \in$ $\mathbb{R}^{n}$ and new matrices $Y_{\theta}, Y_{0}, Y_{1}, Y_{2} \in \mathbb{R}^{n \times n}$

$$
\left\{\begin{array}{l}
\widetilde{\gamma}_{\theta}=\left[\widetilde{J}_{11}, \ldots, \widetilde{J}_{n n}\right]^{T}, \quad Y_{\theta}\left(\dot{v}_{\theta}\right)=\operatorname{diag}\left(\dot{\hat{v}}_{\theta_{1}}, \ldots, \dot{\hat{v}}_{\theta_{n}}\right) \\
\tilde{\gamma}_{0}=\left[\tilde{\Phi}_{0_{11}}, \ldots, \tilde{\Phi}_{0_{n n}}\right]^{T}, Y_{0}\left(\hat{z}_{0}\right)=\operatorname{diag}\left(\hat{z}_{0_{1}}, \ldots, \hat{z}_{0_{n}}\right) \\
\tilde{\gamma}_{1}=\left[\tilde{\Phi}_{1_{11}}, \ldots, \tilde{\Phi}_{1_{n n}}\right]^{T}, Y_{1}\left(\hat{z}_{1}\right)=\operatorname{diag}\left(\hat{z}_{1_{1}}, \ldots, \hat{z}_{1_{n}}\right) \\
\tilde{\gamma}_{2}=\left[\tilde{\Phi}_{2_{11}}, \ldots, \tilde{\Phi}_{2_{n n}}\right]^{T}, Y_{2}\left(v_{\theta}\right)=\operatorname{diag}\left(\hat{v}_{\theta_{1}}, \ldots, \hat{v}_{\theta_{n}}\right) .
\end{array}\right.
$$

The closed loop equation (41) is now rewritten

$$
\begin{aligned}
& J \dot{\hat{\hat{s}}}_{\theta}+K_{I} S(\hat{\varphi})+K_{P} \hat{e}_{\theta}+K_{D} \dot{\hat{\hat{e}}}_{\theta}+A\left(\hat{e}_{\theta}-e_{q}\right) \\
& \quad+K_{S}\left(\dot{\hat{e}}_{\theta}-\dot{e}_{q}\right)-\Phi_{0} \tilde{z}_{0}+\Phi_{1} \Psi \tilde{z}_{1}+\Phi_{2}\left(\dot{\hat{e}}_{\theta}+\Lambda \hat{e}_{\theta}\right) \\
& \quad=Y_{\theta}\left(\dot{\hat{v}}_{\theta}\right) \widetilde{\gamma}_{\theta}+Y_{0}\left(\hat{z}_{0}\right) \tilde{\gamma}_{0}-Y_{1}\left(\hat{z}_{1}\right) \Psi \tilde{\gamma}_{1}+Y_{2}\left(\hat{v}_{\theta}\right) \tilde{\gamma}_{2} .
\end{aligned}
$$

\section{B. Adaptive Computed Torque Control}

For unknown parameters the adaptive control law of the desired link torque $\hat{\tau}_{d}$ is chosen by

$$
\begin{aligned}
\hat{\tau}_{d} & =\widehat{M}(q) \dot{v}_{q}+\widehat{C}(q, \dot{q}) v_{q}+\widehat{g}(q)+K_{q} s_{q} \\
& =Y_{q}\left(q, \dot{q}, v_{q}, \dot{v}_{q}\right) \widehat{\gamma}_{q}+K_{q} s_{q}
\end{aligned}
$$

where $\widehat{M}(q), \widehat{C}(q, \dot{q})$ and $\widehat{g}(q)$ are estimated parameters.

Inserting (44) and (2) into (35) leads to the closed-loop rigid body dynamics $\hat{e}_{\tau}=\hat{\tau}_{d}-\tau$ or

$\hat{e}_{\tau}=-Y_{q}\left(q, \dot{q}, v_{q}, \dot{v}_{q}\right) \widetilde{\gamma}_{q}+M(q) \dot{s}_{q}+\left(C(q, \dot{q})+K_{q}\right) s_{q}$

where $\gamma_{q}, \widehat{\gamma}_{q}$ are the dynamics parameters of the rigid body model and their estimates respectively, and $\widetilde{\gamma}_{q}=\gamma_{q}-\widehat{\gamma}_{q}$.

\section{Stability Analysis}

Theorem 2: For the robot dynamic system (1) and (2) with the adaptive control laws (34), (37), (40) and (44), the controlled system is uniformly stable, and the tracking errors are uniformly bounded and converge to zero $\left\{\lim _{t \rightarrow \infty} \hat{e}_{\theta} \rightarrow 0, \lim _{t \rightarrow \infty} \dot{\hat{e}}_{\theta} \rightarrow 0, \lim _{t \rightarrow \infty} e_{q} \rightarrow\right.$ $\left.0, \lim _{t \rightarrow \infty} \dot{e}_{q} \rightarrow 0, \lim _{t \rightarrow \infty} \tilde{z}_{0} \rightarrow 0, \quad \lim _{t \rightarrow \infty} \tilde{z}_{1} \rightarrow 0\right\}$ whenever condition (25) is fulfilled and the robot parameters are updated by

$$
\left\{\begin{array}{l}
\dot{\hat{\gamma}}_{\theta}=\Gamma_{\theta} Y_{\theta}\left(\dot{\hat{v}}_{\theta}\right) A^{-1} K \hat{s}_{\theta} \\
\dot{\hat{\gamma}}_{q}=\Gamma_{q} Y_{q}\left(q, \dot{q}, v_{q}, \dot{v}_{q}\right) A^{-1} K s_{q} \\
\dot{\hat{\gamma}}_{0}=\Gamma_{0} Y_{0}\left(\hat{z}_{0}\right) A^{-1} K \hat{s}_{\theta} \\
\dot{\hat{\gamma}}_{1}=-\Gamma_{1} \Psi Y_{1}\left(\hat{z}_{1}\right) A^{-1} K \hat{s}_{\theta} \\
\dot{\hat{\gamma}}_{2}=\Gamma_{2} Y_{2}\left(\hat{v}_{\theta}\right) A^{-1} K \hat{s}_{\theta}
\end{array}\right.
$$

with matrices $\Gamma_{\theta}, \Gamma_{q}, \Gamma_{0}, \Gamma_{1}, \Gamma_{2}$ being positive definite and diagonal.

Proof: For stability analysis a new candidate Lyapunov function is selected based on the Lyapunov function $\mathrm{V}$ in (26) and additional terms for the adaptive friction approach

$$
\begin{aligned}
& V_{a}=\frac{1}{2} \hat{s}_{\theta}^{T} K A^{-1} J \hat{s}_{\theta}+\frac{1}{2}\left(\hat{e}_{\theta}-e_{q}\right)^{T} K\left(\hat{e}_{\theta}-e_{q}\right) \\
& +\frac{1}{2} s_{q}^{T} M(q) s_{q}+\frac{1}{2} \hat{e}_{\theta}^{T} K A^{-1}\left(K_{P}+\Lambda K_{D}+\Lambda K_{S}\right) \hat{e}_{\theta} \\
& +e_{q}^{T} K_{q} \Lambda e_{q}+\frac{1}{2} S(\hat{\varphi})^{T} K A^{-1} K_{I} S(\hat{\varphi}) \\
& +\int_{0}^{t}\left(\hat{e}_{\theta}-e_{q}\right)^{T} K \Lambda\left(\hat{e}_{\theta}-e_{q}\right) d t+\frac{1}{2} \widetilde{\gamma}_{q}^{T} \Gamma_{q}^{-1} \widetilde{\gamma}_{q} \\
& +\frac{1}{2} \widetilde{\gamma}_{\theta}^{T} \Gamma_{\theta}^{-1} \widetilde{\gamma}_{\theta}+\frac{1}{2} \tilde{z}_{0}^{T} K A^{-1} \Phi_{0} \tilde{z}_{0}+\frac{1}{2} \tilde{z}_{1}^{T} K A^{-1} \Phi_{1} \Psi \tilde{z}_{1} \\
& +\frac{1}{2} \tilde{\gamma}_{0}^{T} \Gamma_{0}^{-1} \tilde{\gamma}_{0}+\frac{1}{2} \tilde{\gamma}_{1}^{T} \Gamma_{1}^{-1} \tilde{\gamma}_{1}+\frac{1}{2} \tilde{\gamma}_{2}^{T} \Gamma_{2}^{-1} \tilde{\gamma}_{2} .
\end{aligned}
$$


This Lyapunov function $V_{a}$ is always positive definite with positive definite matrices $K_{I}, K_{P}, K_{D}, K_{T}, K_{S}, K_{q}$ and $\Gamma_{\theta}$, $\Gamma_{q}, \Gamma_{0}, \Gamma_{1}, \Gamma_{2} \in \mathbb{R}^{n \times n}$. The derivative of the Lyapunov function $V_{a}$ along the system trajectories is given by

$$
\begin{aligned}
& \dot{V}_{a}=\hat{s}_{\theta}^{T} K A^{-1} J \dot{\hat{s}}_{\theta}+\left(\hat{e}_{\theta}-e_{q}\right)^{T} K\left(\dot{\hat{e}}_{\theta}-\dot{e}_{q}\right) \\
& +\frac{1}{2} s_{q}^{T} \dot{M}(q) s_{q}+s_{q}^{T} M(q) \dot{s}_{q} \\
& +\hat{e}_{\theta}^{T} K A^{-1}\left(K_{P}+\Lambda K_{D}+\Lambda K_{S}\right) \dot{\hat{e}}_{\theta}+2 e_{q}^{T} K_{q} \Lambda \dot{e}_{q} \\
& +\dot{S}(\hat{\varphi})^{T} K A^{-1} K_{I} S(\hat{\varphi})+\left(\hat{e}_{\theta}-e_{q}\right)^{T} K \Lambda\left(\hat{e}_{\theta}-e_{q}\right) \\
& -\dot{\hat{\gamma}}_{q}^{T} \Gamma_{q}^{-1} \widetilde{\gamma}_{q}-\dot{\hat{\gamma}}_{\theta}^{T} \Gamma_{\theta}^{-1} \widetilde{\gamma}_{\theta}+\tilde{z}_{0}^{T} K A^{-1} \Phi_{0} \dot{\tilde{z}}_{0} \\
& +\tilde{z}_{1}^{T} K A^{-1} \Phi_{1} \dot{\tilde{z}}_{1}-\dot{\hat{\gamma}}_{0}^{T} \Gamma_{0}^{-1} \tilde{\gamma}_{0}^{T}-\dot{\hat{\gamma}}_{1}^{T} \Gamma_{1}^{-1} \tilde{\gamma}_{1}^{T}-\dot{\hat{\gamma}}_{2}^{T} \Gamma_{2}^{-1} \tilde{\gamma}_{2}^{T} .
\end{aligned}
$$

By inserting (35), (43), (45) into (48) and utilizing (29), (30) one obtains

$$
\begin{aligned}
& \dot{V}_{a}=\dot{\hat{V}}_{2}-\hat{s}_{\theta}^{T} K A^{-1} \Phi_{2} \hat{s}_{\theta}-\tilde{z}_{0}^{T} K A^{-1} \Phi_{0} \Psi \tilde{z}_{0} \\
& -\tilde{z}_{1}^{T} K A^{-1} \Phi_{1} \Psi \tilde{z}_{1}+\left[\hat{s}_{\theta}^{T} K A^{-1} Y_{\theta}\left(\dot{\hat{v}}_{\theta}\right)-\dot{\tilde{\gamma}}_{\theta}^{T} \Gamma_{\theta}^{-1}\right] \widetilde{\gamma}_{\theta} \\
& +\left[s_{q}^{T} K A^{-1} Y_{q}\left(q, \dot{q}, v_{q}, \dot{v}_{q}\right)-\dot{\tilde{\gamma}}_{q}^{T} \Gamma_{q}^{-1}\right] \widetilde{\gamma}_{q} \\
& +\left[\hat{s}_{\theta}^{T} K A^{-1} Y_{0}\left(\hat{z}_{0}\right)-\dot{\hat{\gamma}}_{0}^{T} \Gamma_{0}^{-1}\right] \tilde{\gamma}_{0}-\left[\hat{s}_{\theta}^{T} K A^{-1} Y_{1}\left(\hat{z}_{1}\right) \Psi\right. \\
& \left.+\dot{\hat{\gamma}}_{1}^{T} \Gamma_{1}^{-1}\right] \tilde{\gamma}_{1}+\left[\hat{s}_{\theta}^{T} K A^{-1} Y_{2}\left(\hat{v}_{\theta}\right)-\dot{\hat{\gamma}}_{2}^{T} \Gamma_{2}^{-1}\right] \tilde{\gamma}_{2} .
\end{aligned}
$$

Analogous to (30), $\dot{\hat{V}}_{2}=\dot{V}_{2}\left(\hat{e}_{\theta}, \dot{\hat{e}}_{\theta}, e_{q}, \dot{e}_{q}\right)$ is negative semidefinite with the condition (25). For negative definiteness of the function $\dot{V}_{a}$ we choose the update law (46) for the dynamics and friction parameters. This leads to

$$
\begin{aligned}
\dot{V}_{a}=\dot{\hat{V}} & -\hat{s}_{\theta}^{T} K A^{-1} \Phi_{2} \hat{s}_{\theta} \\
& -\tilde{z}_{0}^{T} K A^{-1} \Phi_{0} \Psi \tilde{z}_{0}-\tilde{z}_{1}^{T} K A^{-1} \Phi_{1} \Psi \tilde{z}_{1} .
\end{aligned}
$$

Obviously, this function $\dot{V}_{a}$ is negative semi-definite, because all the friction parameters $\Psi, \Phi_{0}, \Phi_{1}, \Phi_{2}$ are positive definite.

Similar to Sec. III, according to Barbalat's lemma it leads to $\left\{\lim _{t \rightarrow \infty} \hat{e}_{\theta} \rightarrow 0, \lim _{t \rightarrow \infty} \dot{\hat{e}}_{\theta} \rightarrow 0, \lim _{t \rightarrow \infty} e_{q} \rightarrow\right.$ $\left.0, \lim _{t \rightarrow \infty} \dot{e}_{q} \rightarrow 0, \lim _{t \rightarrow \infty} \tilde{z}_{0} \rightarrow 0, \lim _{t \rightarrow \infty} \tilde{z}_{1} \rightarrow 0\right\}$, or, equivalently, $\left\{\theta \rightarrow \hat{\theta}_{d}, q \rightarrow q_{d}, \hat{z}_{0} \rightarrow z_{0}, \hat{z}_{1} \rightarrow z_{1}\right\}$ as $t \rightarrow \infty$. This completes the proof of the theorem 2 .

Table I

IDENTIFIED FRICTION PARAMETERS OF THE JOINTS 1-2-3.

\begin{tabular}{lccccc}
\hline \hline Joint & $\begin{array}{c}f_{c} \\
(\mathrm{Nm})\end{array}$ & $f_{l}$ & $\begin{array}{c}f_{v} \\
(\mathrm{Nms} / \mathrm{rad})\end{array}$ & $\begin{array}{c}\sigma_{0} \\
(\mathrm{Nm} / \mathrm{rad})\end{array}$ & $\begin{array}{c}\sigma_{1} \\
(\mathrm{Nms} / \mathrm{rad})\end{array}$ \\
\hline 1 & 2.451 & 0.1263 & 9.2123 & 278.8535 & 28.29 \\
2 & 10.51 & 0.1525 & 14.556 & 5033.382 & 317.6 \\
3 & 10.35 & 0.1432 & 15.484 & 5423.556 & 286.5 \\
\hline
\end{tabular}

Table II

CONTROLLER PARAMETERS FOR THE JOINTS 1-2-3.

\begin{tabular}{lccccccc}
\hline \hline Joint & $k_{I}$ & $k_{P}$ & $k_{D}$ & $k_{T} k^{-1}$ & $k_{S} k^{-1}$ & $k_{q}$ & $\lambda$ \\
\hline 1 & 1619.0 & 8443 & 167 & 1.590 & 0.0068798 & 5 & 10 \\
2 & 1048.4 & 7268 & 301 & 2.966 & 0.0182072 & 5 & 10 \\
3 & 1110.7 & 7714 & 330 & 3.461 & 0.0083539 & 5 & 10 \\
\hline
\end{tabular}

\section{EXPERIMENTS}

In order to validate the control performances, the results of experiments are compared with

1) Controller 1: a PID controller with LuGre-based friction compensation,
2) Controller 2: a proposed cascaded controller with known dynamics parameters using the state feedback controller with integrator terms and LuGre-based friction compensation from section III,

3) Controller 3: a proposed robust adaptive controller with unknown dynamics parameters based on the cascaded control structure from section IV.

All experiments are implemented with joints 1-2-3 of the DLR medical robot. As an example, the tables I, II and III represent the identified friction parameters, the control design parameters and the adaptive parameters of the joints 1-2-3, respectively.

In the proposed control laws the motor velocities and the derivative of the link torques (as well as the link velocities indirectly computed from them) are derived from the measured motor positions and link torques, and then filtered with cutoff frequencies $f_{d \theta}=[200,200,200] \mathrm{Hz}$ and $f_{d \tau}=$ $[100,100,100] \mathrm{Hz}$, respectively.

At first, the control performance in terms of the dynamic (49) behavior of controller 2 from section III is validated by comparing step response results of joint 1 with controller 1 . It can be seen in figure 3 that the proposed controller 2 (the red curve) can superiorly damp oscillations of the link torques.

Furthermore, the robustness of controller 2 is validated against uncertainties of the desired link torques $\tau_{d}$ (dependent on the link dynamics parameters) and the desired friction torques $\tau_{f}$ for friction compensation in the control law (16). By using a periodic trajectory as in figure 4.a, figures 5 and 6 show the motor position errors and measured link torques when the desired link torque $\hat{\tau}_{d}$ is varied from $40 \%$ to $100 \%$ of its identified value $\tau_{d}$ and when the desired friction torque $\hat{\tau}_{f}$ is varied from $0 \%$ to $100 \%$ of its identified value $\tau_{f}$, respectively. It can be seen that the controlled system with the controller 2 is very robust and keeps stability against these disturbances. Based on this robustness the adaptive controller 3 was developed in order to increase position accuracy and reduce the effects of the parameter uncertainties, especially, the high friction effects.

Next, the control performance in terms of the position tracking accuracy (as well as the dynamic behavior) of the proposed controllers is validated. For fast convergence of the estimated parameters in case of the adaptive controller 3 , the initial values of the estimated parameters are set to $100 \%$ of the identified value. Figure 7 shows the adapted friction parameters of the motors 2-3 when they follows the periodic trajectory in figure 4.a. Furthermore, in figure 8 one can see that the adaptive controller 3 (the red curve) with a RMSE value (Root Mean Square Error) $<0.006 \mathrm{deg}$ clearly achieves the best position accuracy in comparison with the controller 1 and 2. Moreover, figure 9 shows the measured link torques to validate the control performance in terms of the dynamic behavior. It can be seen that controller 1 (PID) causes stronger oscillations and thus achieves worse dynamic behavior than

Table III

ADAPTIVE PARAMETERS FOR THE JOINTS 1-2-3.

\begin{tabular}{lccccc}
\hline \hline Joint & $\Gamma_{\theta}$ & $\Gamma_{q}$ & $\Gamma_{0}$ & $\Gamma_{1}$ & $\Gamma_{2}$ \\
\hline 1 & 2 & 2 & 1000 & 1000 & 1000 \\
2 & 2 & 2 & 1000 & 1000 & 1000 \\
3 & 2 & 2 & 1000 & 1000 & 1000
\end{tabular}


the proposed controllers 2 and 3, because its feedback is restricted to motor state variables only, without using link side information such as link position or link torque.

In the next experiment, a point to point trajectory in figure 4.b is chosen in order to show the position tracking accuracy of the robot. Figure 10 shows the motor position accuracy of joints 2-3. It can be seen that the best performance is clearly obtained by the proposed adaptive controller 3 from Sec. IV (the red curve). Whereas controllers 1 and 2 have a steady state error because of the coarsely modeled friction torque and rigid body dynamics, the position error of the adaptive controller 3 quickly converges to zero because of the effects of the integrator and the adaptive compensation.

\section{CONCLUSIONS}

In this paper we proposed a robust cascaded control approach based on using a state feedback control scheme with integrator terms. Further, the method is extended to an adaptive control approach for the case of unknown dynamics parameters in order to enhance the tracking accuracy of flexible joint robot. The control parameters can be simply obtained through the pole placement method in the considered analytical framework. Global asymptotic convergence of the controllers has been proven. Experimental results with the DLR medical robot validate the effectiveness of the proposed controllers in the presence of unknown robot parameters.

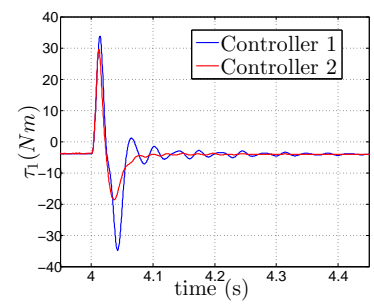

Figure 3. Measured link torque after a step of joint 1 with controller 1 and controller 2
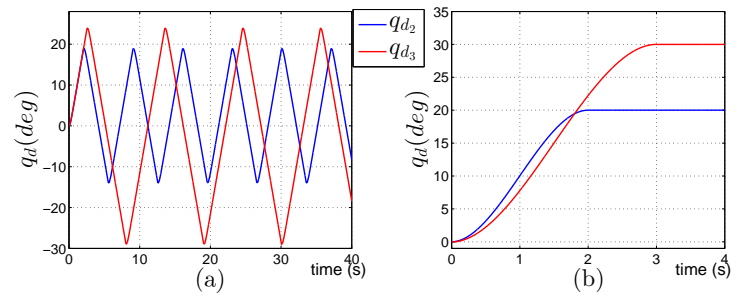

Figure 4. Desired link position for joints 2-3: a) a periodic trajectory; b) a point to point trajectory.

\section{REFERENCES}

[1] M. W. Spong, "Modeling and Control of Elastic Joint Robots", Journal of Dynamic Systems, Measurement and Control, Vol. 109, pp. 310-319, 1987.

[2] H. D. Taghirad, M. A. Khorsavi, "A Robust Linear Controller for Flexible Joint Manipulators", in Proc. IEEE/RSJ Int. Conf. on Intel. Robots and Syst., Sendai, Japan, pp. 2936-2941, 2004

[3] A. De Luca, P. Lucibello, "A General Algorithm for Dynamic Feedback Linearization of Robots with Elastic Joints", in Proc. IEEE Int. Conf. on Rob. and Autom., Leuven, Belgium, pp. 504-510, 1998.

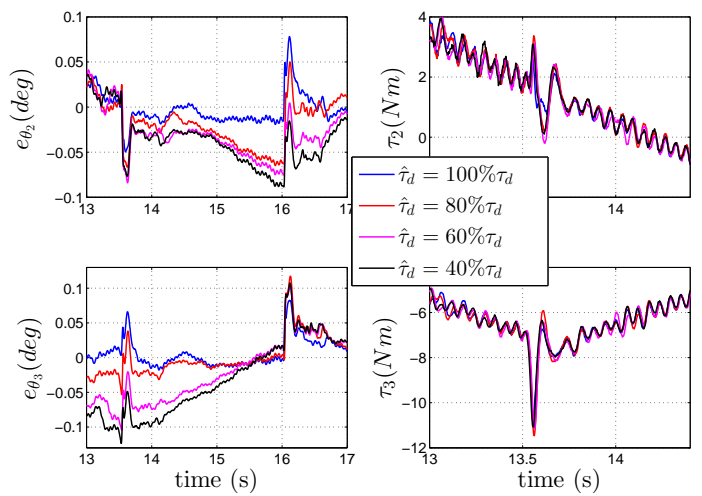

Figure 5. Tracking motor position errors and measured link torques of the proposed controller 2 during the periodic trajectory when the desired link torque varies.

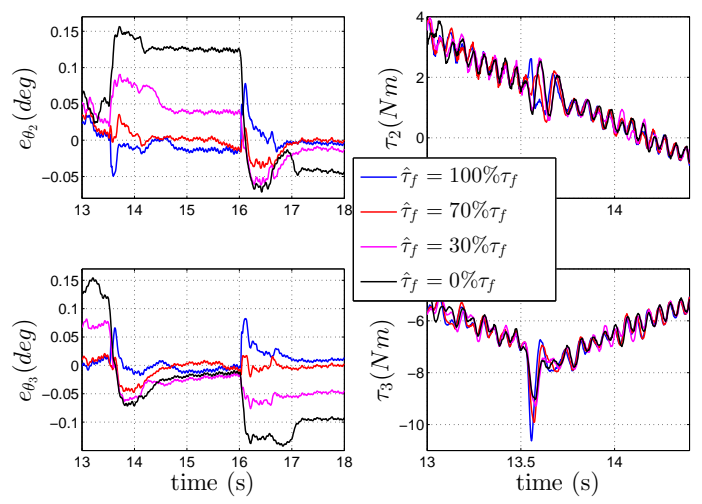

Figure 6. Tracking motor position errors and measured link torques of the proposed controller 2 during the periodic trajectory when the desired friction torque varies.

[4] S. Moberg, S. Hanssen, "On Feedback Linearization for Robust Tracking Control of Flexible Joint Robots", The Int. Federation of Automatic Control, pp. 12218-12223, 2008

[5] L. Tian, A. A. Goldenberg, "Robust Adaptive Control of Flexible Joint Robots with Joint Torque Feedback", in Proc. IEEE Int. Conf. on Rob. and Autom., Nagoya, Japan, pp. 1229-1234, 1995.

[6] M. M. Fateh, "Robust Control of Flexible-Joint Robot Using Voltage Control Strategy", Nonlinear Dynamics, pp. 1525-1537, 2012.

[7] B. Brogliato, R. Ortega, R. Lozano, "Global Tracking Controllers for Flexible-Joint Manipulators: a Comparative Study", Automatica, Vol. 31, Issue 7, pp. 941-956, 1995.

[8] J. Lee, J .S. Yeon, J. H. Park, S. Lee, "Robust Back-Stepping Control for Flexible-Joint Robot Manipulators", in Proc. IEEE/RSJ Int. Conf. on Intel. Rob. and Syst., San Diego, USA, pp. 183-188, 2007.

[9] C. Ott, A. Albu-Schäffer, A. Kugi, G. Hirzinger, "On the passivitybased impedance control of flexible joint robots", IEEE Trans. on Rob., Vol. 24, pp. 416-429, 2008.

[10] C.-I. Morarescu, B. Brogliato, "Passivity-based switching control of flexible-joint complementarity mechanical systems", Automatica, Vol. 46 Issue 1, pp. 160-166, 2010.

[11] K. Melhem, W. Wang, "Global output tracking control of flexible joint robots via factorization of the manipulator mass matrix", IEEE Trans. on Rob., Vol. 25, Iss.2, pp. 428-437, 2009.

[12] S. Ibrir, "Nonlinear observer design with robustness of transient behavior: Application to a flexible-joint robot", in Proc. IEEE Int. Conf. on Cont. Appl. Part of 2010 IEEE Multi-Conf. on Syst. and Cont., Yokohama, Japan, pp. 1385-1390, 2010.

[13] J. Lee, T. J. Hal, J. S. Yeon, S. Lee, J. H. Park, "Robust Nonlinear Observer for Flexible Joint Robot Manipulators with Only Motor Position Measurement", in Proc. Int. Conf. on Cont., Autom. and Syst., Seoul, Korea, pp. 17-20, 2007.

[14] A. Albu-Schäffer, C. Ott, G. Hirzinger, "A unified passivity-based control framework for position, torque and impedance control of flexible joint robots", The Int. Journal of Rob. Research, pp. 23-39, 2007. 

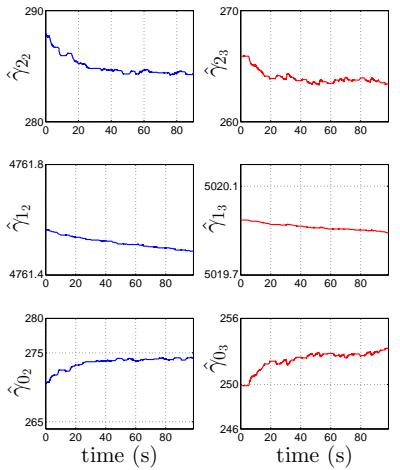

Figure 7. Estimated friction parameters of joints 2-3 from the adaptive controller 3 during the periodic trajectory.

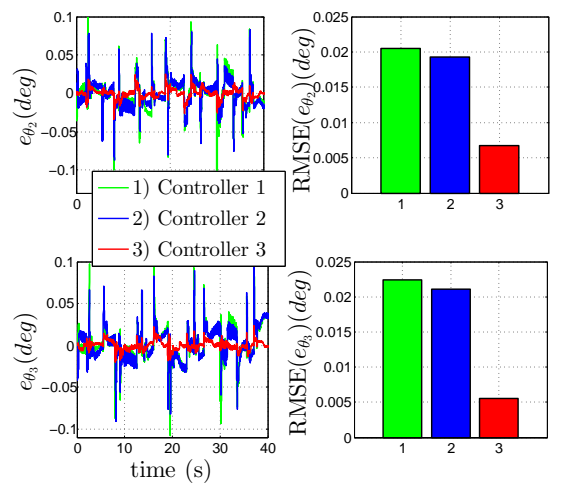

Figure 8. Tracking motor position errors and their RMSE (Root Mean Square Error) during the periodic trajectory with controller 1, controller 2 and controller 3 .

[15] L. Le-Tien, A. Albu-Schäffer, "Improving Tracking Accuracy of a MIMO State Feedback Controller for Elastic Joint Robots", in Proc. 53rd IEEE Conf. on Dec. and Cont., Los Angeles, USA, pp. 4548-4553, 2014.

[16] C. Kwan, K. S. Yeung, "Robust adaptive control of revolute flexiblejoint manipulators using sliding technique", Syst. \& Cont. Letters, Vol. 20, Iss. 4, pp. 279-288, 1993.

[17] W. H. Zhu, E. Dupuis, M. Doyon, "Adaptive Control of Harmonic Drives", Trans. of the ASME, pp. 182-193, 2007.

[18] L. Le-Tien, A. Albu-Schäffer, "Adaptive Friction Compensation in Trajectory Tracking Control of DLR Medical Robots with Elastic Joints", in Proc. IEEE/RSJ Int. Conf. on Intel. Rob. and Syst., Vilamoura, Algarve, Portugal, pp. 1149-1154, 2012.

[19] C. C. de Wit, P. Linschinsky, "Adaptive friction compensation with partially known dynamic friction model", Int. Journal of Adap. Cont. and Sig. Processing, Vol. 11, pp. 65-80, 1997.

[20] P. Tomei, "Robust Adaptive Friction Compensation for Tracking Control of Robot Manipulators", IEEE Trans. on Rob. and Autom., pp. 2164-2169, 2000.

[21] Q. H. Xia, S. Y. Lim, M. H. A. Jr, T. M. Lim, "Adaptive Joint Friction Compensation Using a Model-Based Operational Space Velocity Observer", in Proc. IEEE Int. Conf. on Rob. and Autom., pp. 3081-3086, 2004.

[22] R. Ortega, M. W. Spong, "Adaptive Motion Control of Rigid Robots: A Tutorial", in Proc. Conf. on Dec. and Cont., pp. 1575-1584, 1988.

[23] U. Hagn, M. Nickl, S. Jörg, G. Passig, T. Bahls, A. Nothhelfer, F. Hacker, L. Le-Tien, A. Albu-Schäffer, R. Konietschke, M. Grebenstein, R. Warpup, R. Haslinger, M. Frommberger, G. Hirzinger, "The DLR MIRO: A versatile lightweight robot for surgical applications", Industrial Robot: An International Journal, Vol.35, pp. 324-336, 2008.

[24] A. De Luca, P. Tomei, "Elastic Joints in C. Canudas de Wit, B. Siciliano, G. Bastin (Eds.): Theory of Robot Control", pp. 179-217, Springer London, 1996.

[25] C. C. de Wit, H. Olsson, K. J. Astron, P. Linschinsky, ”A New Model for Control of Systems with Friction", IEEE Trans. on Aut. Cont., Vol. 40, pp. 419-425, 1994.

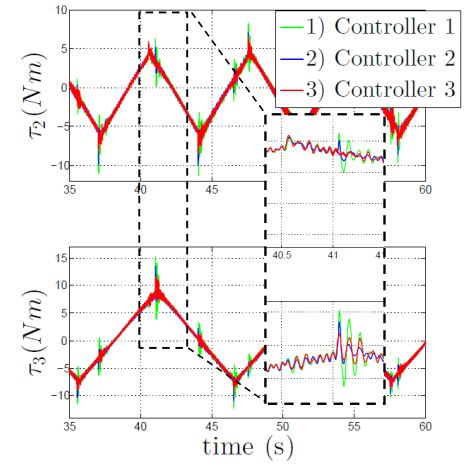

Figure 9. Measured link torque during the periodic trajectory with controller 1 , controller 2 and controller 3 .

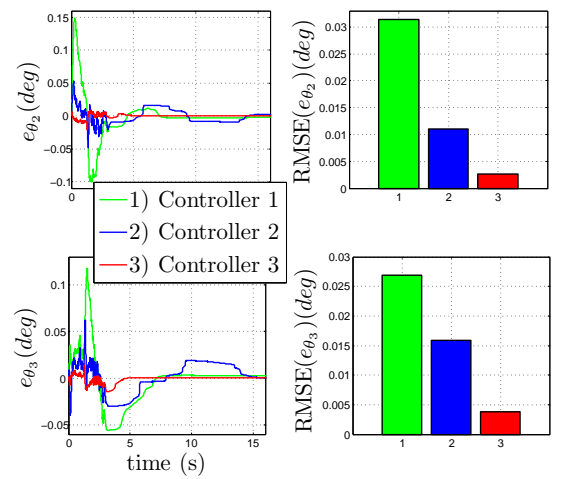

Figure 10. Tracking motor position errors and their RMSE during the point to point trajectory with controller 1 , controller 2 and controller 3 .

[26] J-J. E. Slotine, W. Li, "Applied Nonlinear Control", New Jersey, Englewood Cliffs: Prentice Hall, 1991.

[27] Y. Tan, I. Kanellakopoulos, "Adaptive Nonlinear Friction Compensation with Parametric Uncertainties", in Proc. of American Control Conference, pp. 2511-2515, 1999.

[28] S. Boyd, L. Vandenberghe, "Convex Optimization", Cambridge University Press, 2004.

Luc Le-Tien Luc Le-Tien received his B.S. and M.S. in electrical engineering from the Hanoi University of Science and Technology, Vietnam in 1996 and 1999, respectively, and his Ph.D. in electrical and computer engineering from the Technical University of Dresden in 2010. From 2000 to 2002 he worked at the Hanoi University of Science and Technology, Vietnam. He is currently a research scientist at the Institute of Robotics and Mechatronics at the German Aerospace Center (DLR), which he joined in 2002 as a Ph.D. candidate. His current research interests include modeling, identification and control, nonlinear control, adaptive control, tracking control, friction compensation, impedance and admittance control, flexible joint robots, floating base robots, medical robots. 
Alin Albu-Schäffer Alin Albu-Schäffer received his M.S. in electrical engineering from the Technical University of Timisoara, Romania in 1993 and his $\mathrm{Ph} . \mathrm{D}$. in automatic control from the Technical University of Munich in 2002. Since 2012 he is the head of the Institute of Robotics and Mechatronics at the German Aerospace Center (DLR), which he joined in 1995 as a Ph.D. candidate. Moreover, he is a professor at the Technical University of Munich, holding the Chair for "Sensor Based Robotic Systems and Intelligent Assistance Systems" at the Computer Science Department. His personal research interests include robot design, modeling and control, nonlinear control, flexible joint and variable compliance robots, impedance and force control, physical human-robot interaction, bio-inspired robot design and control. He received several awards, including the IEEE King-Sun Fu Best Paper Award of the Transactions on Robotics in 2012, several ICRA and IROS Best-Paper-Awards as well as the DLR Science Award. 